\title{
The Impact of Communication Skills Training in Oncology: a Linguistic Analysis
}

\author{
Pascal Singy • Céline Bourquin • Brikela Sulstarova • \\ Friedrich Stiefel
}

Published online: 21 June 2012

(C) Springer Science+Business Media, LLC 2012

\begin{abstract}
This study aimed to investigate the impact of a communication skills training (CST) in oncology on clinicians' linguistic strategies. A verbal communication analysis software (Logiciel d'Analyse de la Communication Verbale) was used to compare simulated patients interviews with oncology clinicians who participated in CST $(N=57)$ (pre/ post with a 6-month interval) with a control group of oncology clinicians who did not $(N=56)(\mathrm{T} 1 / \mathrm{T} 2$ with a 6-month interval). A significant improvement of linguistic strategies related to biomedical, psychological and social issues was observed. Analysis of linguistic aspects of videotaped interviews might become in the future a part of individualised feedback in CST and utilised as a marker for an evaluation of training.
\end{abstract}

Keywords Cancer · Clinician-patient communication · Communication skills training $\cdot$ Oncology $\cdot$ Linguistic strategies

\section{Introduction}

Over the last decade, there has been an increase of so called communication skills trainings (CSTs). CST is particularly relevant for oncology, where threatening and complex information is communicated and crucial decisions have to be made [1-4]. It has been observed (1) that ineffective communication increases patients'

P. Singy $(\bowtie) \cdot$ C. Bourquin $\cdot$ B. Sulstarova $\cdot$ F. Stiefel Psychiatric Liaison Service, Lausanne University Hospital, Les Allières,

1011 Lausanne-CHUV, Switzerland

e-mail: pascal.singy@chuv.ch confusion [5] and clinicians' dissatisfaction [6] and (2) that oncology clinicians tend to adopt strategies, which result on a linguistic level in an excessive use of implicit formulations [7], medical jargon [8], evasive language $[1,7,8]$ and a rigid interactional setting (e.g. directivity of questions) [9-11]. On the other hand, CST has been demonstrated to be effective with regard to physicians' and patients' outcomes, such as clinicians' skill improvement or patients' information recall, adherence to treatment and pain control [12].

Different approaches to evaluate CST have been utilised focusing on various aspects [12-17], including clinicians' defence mechanisms [15] or patient-clinician interactions [16]. As recently stated in a European consensus paper [17], research should continue to evaluate CST from different perspectives. This study complements traditional methods to evaluate CST by assessing clinicians' linguistic strategies by means of a software (Logiciel d'Analyse de la Communication Verbale (LaComm), www.lacomm.be) that analyses the linguistic content of medical communication, based on a similar type of procedure as the frequently used Roter interaction analysis system (RIAS), which is designed as a rating system by an observer. In contrast, LaComm provides a computational rating. It distinguishes between three main functions of an (oncology) consultation: assessing, supporting and informing the patient [18]. Each of these functions is based on specific communicational skills: (1) assessment skills used when clinicians invite patients to report their physical and psychosocial concerns, (2) support skills used when they empathically respond to patients' concerns and needs and (3) information skills used when they structure the interview and provide information about the disease and its treatments. 


\section{Methods}

The study-based on a two-group pre/post controlled trial - evaluated interviews of oncology clinicians who participated in a CST (CST group) and oncology clinicians who did not (control (CTRL) group). The interviews were first analysed with regard to clinicians' defence mechanisms, demonstrating that defences change under training with an increase of mature defences in the CST group for clinicians with a higher initial overall defensive functioning [15]. A second grant was obtained to analyse this material from a linguistic point of view.

\section{Sample}

The CST group, enrolled between 2000 and 2006, consisted of 57 French-speaking oncology physicians $(N=30)$ and nurses $(N=27)$ of whom $43(75.4 \%)$ were women and 14 $(24.6 \%)$ were men with a mean age of 37.9 years $(\mathrm{SD}=7.2)$. The CTRL group, enrolled between 2004 and 2006, consisted of 56 French-speaking oncology physicians $(N=21)$ and nurses $(N=35)$ of whom $35(62.5 \%)$ were women and $21(37.5 \%)$ were men with a mean age of 39.4 years $(\mathrm{SD}=9.3)$ (no significant differences between groups).

\section{Intervention (CST)}

The CST consisted of a 2-day course, followed by four to six individual supervisions and a half-day training 6 months later [19]. A maximum of ten oncology clinicians (physicians and nurses) participate in a given CST, which is based on video feedback of interviews with simulated patients, role play and interactive case presentations, focusing on four major aspects of communication: structure of the interview (time, space, participants, negotiation of the patient's and clinician's agenda, transitions to new topics, intermediate syntheses, etc.), exchange of information (different types of questions, adequacy of language, amount of information provided, etc.), emotions (verbal and non-verbal expressions of the patient's emotion, identification of emotional expression, empathic responses, etc.) and relational aspects (encouraging the patient to express his views and concerns, clarifying his needs, maintaining narrativity, etc.).

Each participant of the CST conducted two videotaped interviews of $15 \mathrm{~min}$ with a simulated patient (professional actors experienced in playing cancer patients), one before training and one 6 months later. Prior to the interviews, both the oncology clinicians and the actors received written instructions specifying the type of cancer (breast, stomach, testicles and lymphoma) and its treatment (curative or palliative) and the purpose of the interview (announcing the diagnosis of a curable cancer or proposition of palliative treatment for physicians; preparing the patient for curative or palliative chemotherapy for nurses). Participants of the CTRL group also conducted two videotaped interviews of 15 min with a 6-month interval based on the same scenarios and with the same actors (simulated patients).

Assessment: Verbal Communication Analysis (LaComm)

LaComm is a content analysis software, developed by Razavi et al. [20] for the linguistic assessment of medical communication. LaComm is based on databases which are similar to those of softwares like Protan, LIWC or General Inquirer.

The software performs, in a single-stage process, a twofold analysis of clinicians' discourse and of patients' discourse. Discourse of clinicians is analysed (1) by identifying utterances reflecting different communication strategies, which are regarded as sets of statements produced in order to achieve a communication goal and (2) by classifying the words of these utterances, depending on their meaning, in categories of distinct topics. Given that no specific strategies are expected from the patients, their discourses are only classified in topic categories, which means that only words are taken into account.

Clinicians' utterances are classified in three categories of communication strategies corresponding to the abovementioned functions of an oncology consultation: (1) assessment, (2) support and (3) information of the patient. Each of these categories is further divided into several subcategories (see Table 1). The assessment strategy category includes utterances which allow clinicians to obtain information about the patient's disease and his physical and psychosocial concerns (e.g. "did you begin the treatment?", subcategory "closed binary assessment"). The support strategy category includes utterances which allow clinicians to acknowledge patient's concerns, to reassure him and to take into account his opinions (e.g. "I understand", subcategory "basic hetero-centred acknowledgment"). The information strategy category includes utterances which allow clinicians to inform about the context of the consultation and the planned examination (e.g. "I am Doctor $\mathrm{X}$ ", subcategory "context of the interview").

Words of clinicians and patients - related to specific topics - are classified into three topic categories: (a) medical topic, (b) psychological topic and (c) social topic. Each category is again further divided into several subcategories (see Table 1). The medical topic category includes words belonging to subcategories, such as "precise oncological diagnosis" (e.g. cancer, Hodgkin's), "imprecise oncological diagnosis" (e.g. anomaly, illness) or "treatments, techniques and similar words" (e.g. morphine, 
Table 1 Examples of LaComm's categories

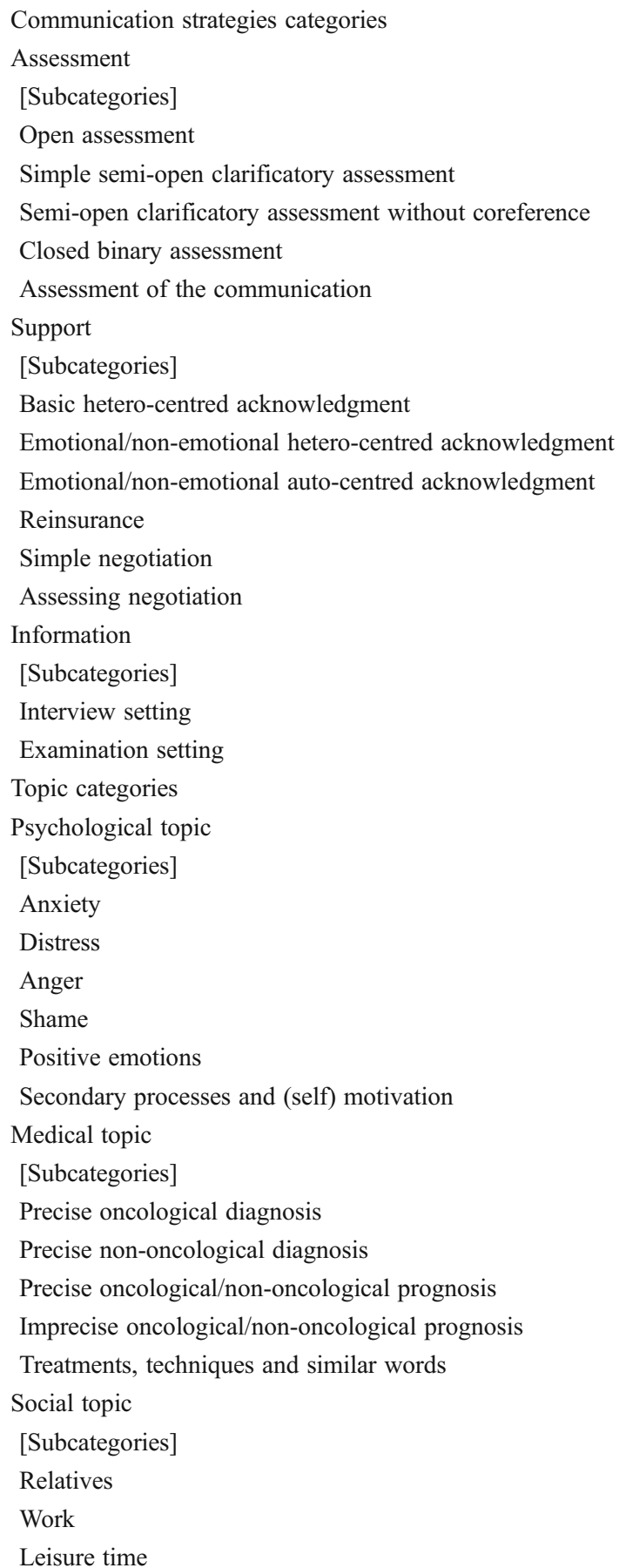

Leisure time

catheter). The psychological topic category includes words belonging to subcategories, such as "anxiety" (e.g. afraid, nervous) or "distress" (e.g. sad, helplessness). And the social topic category includes words relating to subcategories, such as "close relations" (e.g. family, wife), "work" (e.g. job, salary) or "leisure time" (e.g. activities, hobbies).
Data Analyses

Since some subcategories-for example words referring to clothes-were not considered to be relevant within the scope of the study and others were not used, only 44 of the 81 subcategories of LaComm (see www.lacomm.be) were included in the analysis. The results of each of these 44 subcategories were compared between CST and CTRL groups. ANOVA were performed with LaComm's subcategories as dependent variables. The variance analysis was computed by the statistical software SPSS 15.0 (for Windows). Cohen's $d$ effect size measurements were computed for the ANOVA and the $t$ tests. All tests were two-tailed and the alpha was set at .05

\section{Results}

LaComm's Mean Frequencies for the CST and the CTRL Group

For both the CST and the CTRL groups, the three most frequent subcategories of utterances and words (19 to 27 occurrences per interview) were treatments, techniques and similar words, basic hetero-centred acknowledgment and secondary processes and (self) motivation (see Table 2). The subcategory treatments, techniques and similar words includes words referring to medical equipment and treatments and/or medical procedures (e.g. names of medication). The subcategory basic hetero-centred acknowledgment includes utterances used to encourage the patient to express himself (e.g. "I understand you"). The subcategory secondary processes and (self) motivation includes words referring to cognitive processes (e.g. to decide) or to the personality (e.g. courage). Fourteen other subcategories had a mean frequency of 2 to 6 and 27 subcategories had only a mean frequency of 0.02 to 2 occurrences per interview and were therefore not included in the analysis.

\section{Changes After CST}

While no change between time 1 and time 2 was observed for the CST and the CTRL groups with regard to the utterances, statistically significant differences were observed with regard to the identified words (see Table 3). The differences occurred in all topic categories (medical, psychological and social). After CST, clinicians used more often words related to the following subcategories: in the medical topic category for the subcategory precise oncological diagnosis $(p=.049)$; in the psychological topic category for the subcategory secondary processes and (self) motivation $(p=.011)$ and in the social topic category for the subcategories 
Table 2 LaComm's subcategories with the highest mean

\begin{tabular}{|c|c|c|c|c|c|c|c|c|}
\hline & \multicolumn{4}{|l|}{ CST } & \multicolumn{4}{|l|}{ CTRL } \\
\hline & \multicolumn{2}{|l|}{ Pre } & \multicolumn{2}{|l|}{ Post } & \multicolumn{2}{|l|}{$\mathrm{T} 1$} & \multicolumn{2}{|l|}{$\mathrm{T} 2$} \\
\hline & M & SD & M & SD & M & SD & M & SD \\
\hline Treatments, techniques and similar words & 25.70 & 12.546 & 27.35 & 13.182 & 25.20 & 13.455 & 23.84 & 13.222 \\
\hline Basic hetero-centred acknowledgment & 26.26 & 14.589 & 26.74 & 14.211 & 23.59 & 14.596 & 21.25 & 14.737 \\
\hline Secondary processes and (self) motivation* & 19.86 & 7.340 & 23.86 & 9.156 & 21.91 & 10.342 & 19.46 & 10.441 \\
\hline
\end{tabular}

CST group with the communication skills training $(N=57), C T R L$ group without the communication skills training $(N=56)$

$* p=.011$

work $(p=.048)$ and leisure time $(p=.001)$. Group contrasts effect sizes were small to medium.

\section{Discussion}

Compared to the CTRL group, clinicians who benefited from CST showed no changes with regard to the analysis of utterances, but an increase of words in the categories indicating the medical, psychological and social topics. The fact that changes after CST are observable only on the level of words (or topics investigated) and not with regard to utterances (or strategies of communication) is not surprising. With other words, CST did not lead clinicians to change the dynamics of their interview, for example by changing the proportion of communication focusing on assessment, support and information provided. However, words investigating certain topics increased, illustrating that clinicians more often used precise diagnostic terms, such as "carcinoma" or "malignant" (subcategory "precise oncological diagnosis") and thus relatively fewer terms which are vague, such as "nodules" or "cells". Whether these changes represent a positive development (accurate information of the patient) or not (use of jargon) remains unanswered. However, what is often observed is that participants use avoidance strategies before training and are then enabled by CST to name the medical problem by its name, since they feel more comfortable to speak about difficult issues. Depending on the patient's desire to be informed, the fact that a clinician feels secure to face threatening news seems to be beneficial; on the contrary, anxiety is often contagious and distressing, also between patients and clinicians.

This hypothesis that CST improved linguistic aspects of communication is also confirmed by an increase of words related to the subcategory secondary processes and self motivation, which represents the patient's experiences of the illness (e.g. "impressions" (of the patient)), as well as the subcategory of words indicating work (e.g. "profession") and leisure-related issues (e.g. "family"), thus giving room to the patient's subjectivity and fostering the therapeutic alliance.

While a linguistic analysis does not provide an evaluation of the quality of a given interview, it provides indicators of

Table 3 Changes between pre/post and T1/T2 in LaComm's subcategories for the CST and CTRL groups

\begin{tabular}{|c|c|c|c|c|c|c|c|c|c|c|c|c|c|c|}
\hline & \multicolumn{7}{|l|}{$\mathrm{CST}$} & \multicolumn{7}{|l|}{ CTRL } \\
\hline & \multicolumn{2}{|l|}{ Pre } & \multicolumn{2}{|l|}{ Post } & \multirow[b]{2}{*}{$F$} & \multirow[b]{2}{*}{$p$} & \multirow[b]{2}{*}{$d$} & \multicolumn{2}{|l|}{$\mathrm{T} 1$} & \multicolumn{2}{|l|}{$\mathrm{T} 2$} & \multirow[b]{2}{*}{$F$} & \multirow[b]{2}{*}{$p$} & \multirow[b]{2}{*}{$d$} \\
\hline & M & SD & M & SD & & & & M & SD & M & SD & & & \\
\hline $\begin{array}{l}\text { Precise } \\
\text { oncological } \\
\text { diagnosis }\end{array}$ & 2.49 & 3.790 & 4.49 & 6.577 & 3.957 & .049 & -0.37 & 3.75 & 5.428 & 3.18 & 4.605 & 0.361 & .549 & 0.11 \\
\hline $\begin{array}{l}\text { Secondary } \\
\text { processes and } \\
\text { (self) } \\
\text { motivation }\end{array}$ & 19.86 & 7.340 & 23.86 & 9.156 & 6.623 & .011 & -0.48 & 21.91 & 10.342 & 19.46 & 10.441 & 1.552 & .216 & 0.23 \\
\hline Work & 2.05 & 2.271 & 2.98 & 2.676 & 4.001 & .048 & -0.37 & 2.46 & 2.683 & 2.96 & 3.547 & 0.708 & .402 & -0.15 \\
\hline Leisure time & 0.42 & 0.755 & 1.11 & 1.319 & 11.560 & .001 & -0.64 & 0.95 & 1.600 & 1.07 & 1.548 & 0.177 & .675 & -0.07 \\
\hline
\end{tabular}

CST group with the communication skills training $(N=57), C T R L$ group without the communication skills training $(N=56) p \leq .05, F$ between-group variance/within-group variance, $d$ effect size measure 
how balanced or unbalanced an interview may be from a linguistic point of view. Such markers might be useful for an individual feedback for participants of CST or for research. Up to now, methods evaluating the impact of CST were often based on observer-rated instruments, such as the RIAS. It would therefore be important to have linguistic markers which can be tailored to the objectives of a given CST and identified by a software analysis. LaComm is certainly still a quite complicated method, since interviews have to be transcribed first, but it may represent a first step towards new ways of efficiently analysing medical interviews. In the future, such analyses should be based on audio-analyses which do not depend on transcription of interviews.

Acknowledgments The study was supported by OncoSuisse, grant 02035-02-2007. We would like to thank Isaac Pante for assisting us with the data analyses.

Conflict of Interest The authors declare that they have no conflict of interest.

\section{References}

1. Lutfey K, Maynard DW (1998) Bad news in oncology: how physician and patient talk about death and dying without using those words. Soc Psychol Quart 61(4):321-341

2. Ptacek JT, Fries EA, Eberhardt TL (1999) Breaking bad news to patients: physicians' perceptions of the process. Support Care Cancer 7:113-120

3. Fallowfield L, Jenkins V (2004) Communicating sad, bad and difficult news in medicine. Lancet 363:312-319

4. Siefel F, Razavi D (2006) Informing about diagnosis, relapse and progression of disease-communication with terminally ill cancer patient. In: Stiefel F (ed) Communication in cancer care. Springer, Berlin, pp 37-46

5. Lerman C, Daly M, Walsh WP et al (1993) Communication between patients with breast cancer and health care providers: determinants and implications. Cancer 72:2612-2620
6. Fallowfield L (1995) Can we improve the professional and personal fulfilment of doctors in cancer medicine? Br J Cancer 71:1132-1133

7. Beisecker AE, Beisecker TD (1993) Using metaphors to characterize doctor-patient relationships: paternalism versus consumerism. Health Commun 5(1):41-58

8. Barton E (2004) Discourse methods and critical praxis in professional communication. J Bus Tech Commun 18(1):67-111

9. Beach WA, Easter DW, Good JS, Pigeron E (2005) Disclosing and responding to cancer fears during oncology interviews. Soc Sci Med 60:893-910

10. Collins S, Drew P, Watt I, Entwistle V (2005) 'Unilateral' and 'bilateral' practitioner approaches in decision-making about treatment. Soc Sci Med 61:2611-2627

11. Libert Y, Janne P, Razavi D et al (2003) Impact of medical specialists' locus of control on communication skills in oncological interviews. Br J Cancer 88:502-509

12. Barth J, Lannen P (2011) Efficacy of communication skills training courses in oncology: a systematic review and meta-analysis. Ann Oncol 22(5):1030-1040

13. Gysels M, Richardson A, Higginson IJ (2005) Communication training for health professionals who care for patients with cancer: a systematic review of training methods. Support Care Cancer 13:356-366

14. Supiot S, Bonnaud-Antignac A (2008) Using simulated interviews to teach junior medical students to disclose the diagnosis of cancer. J Cancer Educ 23:102-107

15. Bernard M, Roten Y, Despland JN, Stiefel F (2010) Communication skills training and clinicians' defenses in oncology: an exploratory, controlled study. Psycho-Oncology 19:209-215

16. Langewitz W, Heydrich L, Nubling M et al (2010) Swiss Cancer League communication skills training programme for oncology nurses: an evaluation. JAN 66(10):2266-2277

17. Stiefel F, Barth J, Bensing J et al (2010) Communication skills training in oncology: a position paper based on a consensus meeting among European experts in 2009. Ann Oncol 21:204-207

18. Razavi D, Delvaux N (2008) Précis de psycho-oncologie. Masson, Paris

19. Stiefel F, Bernhard J, Bianchi G et al (2010) The Swiss model. In: Kissane D, Bultz B, Butow P, Finlay I (eds) Handbook of communication in oncology and palliative care. Oxford University Press, Oxford, pp 641-648

20. Liénard A, Merckaert I, Libert $Y$ et al (2010) Is it possible to improve residents breaking bad news skills? A randomised study assessing the efficacy of a communication skills training program. Br J Cancer 103:171-177 